\title{
Bone Mesenchymal Stem Cell-Derived sEV-Encapsulated Thermosensitive Hydrogels Accelerate Osteogenesis and Angiogenesis by Release of Exosomal miR-21
}

\section{OPEN ACCESS}

Edited by:

Zongliang Wang,

Changchun Institute of Applied

Chemistry (CAS), China

Reviewed by:

Pingping Han,

The University of Queensland, Australia

Xiaoxiao Cai,

Sichuan University, China

*Correspondence:

Xiaojuan Wei

xjweish@126.com

Changqing Zhang

zhangcq@sjtu.edu.cn

Leilei Liang

liangleilei10006@163.com

${ }^{\text {t}}$ These authors have contributed equally to this work

Specialty section: This article was submitted to Biomaterials,

a section of the journal Frontiers in Bioengineering and

Biotechnology

Received: 05 December 2021 Accepted: 27 December 2021

Published: 19 January 2022

Citation:

Wu D, Qin H, Wang Z, Yu M, Liu Z, Peng $H$, Liang L, Zhang $C$ and Wei $X$ (2022) Bone Mesenchymal Stem Cell-

Derived SEV-Encapsulated

Thermosensitive Hydrogels Accelerate Osteogenesis and Angiogenesis by

Release of Exosomal miR-21.

Front. Bioeng. Biotechnol. 9:829136.

doi: 10.3389/fbioe.2021.829136

\author{
Di Wu ${ }^{1 \dagger}$, Hao Qin ${ }^{2 \dagger}$, Zixuan Wang ${ }^{3 \dagger}$, Mingzhao Yu ${ }^{1}$, Zhe Liu ${ }^{1}$, Hao Peng ${ }^{1}$, Leilei Liang ${ }^{4 *}$, \\ Changqing Zhang ${ }^{1 *}$ and Xiaojuan Wei ${ }^{5 *}$
}

\begin{abstract}
'Department of Orthopedic Surgery, Shanghai Jiao Tong University Affiliated Shanghai Sixth People's Hospital, Shanghai, China, ${ }^{2}$ Center for Reproductive Medicine, Department of Obstetrics and Gynecology, Peking University Third Hospital, Beijing, China, ${ }^{3}$ Department of Mechanical Engineering, Tsinghua University, Beijing, China, ${ }^{4}$ National Cancer Center, Chinese Academy of Medical Sciences and Peking Union Medical College, Beijing, China, ${ }^{5}$ Institute of Microsurgery on Extremities, Shanghai Jiao Tong University Affiliated, Shanghai Sixth People's Hospital, Shanghai, China
\end{abstract}

Angiogenesis has been recognized to play an essential role in remodeling new bone (osteogenesis). Small extracellular vesicles (sEVs), the endogenously secreted nanovesicles by cells, exhibit great potential in the regeneration of bone defects and the realization of cell-free therapy. Chitosan, a natural polysaccharide, can form a thermosensitive injectable hydrogel through the addition of $\beta$-glycerophosphate. Herein, we developed injectable thermosensitive hydrogel-encapsulated sEVs derived from bone mesenchymal stem cells, which significantly prolonged delivery and release and synergistically enhanced bone regeneration. sEVs were isolated and characterized, and the physicochemical properties, release kinetics, and biocompatibility of the hydrogels were analyzed. In vitro experiments were performed to investigate osteogenic differentiation, cell proliferation and migration, and tube formation. Thereafter, sEVs were added to the chitosan/ $\beta$-glycerophosphate hydrogel (sEV@CS/ $/$-GP composite) to repair calvarial defects in rats. The results showed that sEV-loaded hydrogels were biocompatible, exhibiting excellent thermosensitive properties and enhancing bone regeneration. Furthermore, mechanistic studies revealed that exosomal miR-21 targeted SPRY2, thereby promoting angiogenesis. Our study provides new insights on the repair of bone defects with multifunctional controlled-sEV-release hydrogels, which shows great potential in the repair of tissues in the future.

Keywords: thermosensitive hydrogel, small extracellular vesicle, release, bone regeneration, angiogenesis

\section{INTRODUCTION}

Bone defects often arise from trauma, infections, tumors, congenital malformations, or skeletal diseases, and they can heal slowly or not at all, thereby leading to life-long disabilities (Zaidi, 2007; Huey et al., 2012). Currently, the "gold standard" in the treatment of bone defects is autologous and allogeneic transplantation (Crane et al., 1995; Lopes et al., 2018). In recent years, the introduction of a variety of reconstructive materials has provided new tools for orthopedic surgeons; however, the 
most important aspect of bone regeneration still lies in the identification of safe, effective, and aesthetic means for filling structural defects (Kawai et al., 2011; Bose et al., 2012; Zhang et al., 2019). Several studies on the modulatory processes of bone healing have demonstrated that angiogenesis plays an important role in bone regeneration. As such, bone tissue engineering combines the use of different cells, biological factors, scaffolds, and bone substitutes to improve osteogenic and angiogenic activities (Langer and Vacanti, 1993; Khademhosseini and Langer, 2016).

The use of bone mesenchymal stem cells (BMSCs) is an attractive approach to promote osteogenesis and angiogenesis in patients with bone defects (Fu et al., 2019). BMSCs can be easily harvested from donors, maintain osteogenic properties, and offer a low incidence of graft-versus-host disease. However, the direct transplantation of BMSCs associates with several challenges such as time- and dose-requirements, the low survival rate of locally transplanted cells, tumor formation, and immune rejection (Sissung and Figg, 2020). In addition, the role of BMSCs in tissue regeneration involves paracrine mechanisms, which stimulate immunomodulatory pathways, and small extracellular vesicles (sEVs) have been implicated in these processes (Li et al., 2018).

sEVs are membranous structures (diameter, 50-150 nm), namely, exosomes and microvesicles that are released from cells into the extracellular environment, and they participate in cell-to-cell communication (Tkach and Thery, 2016; van Niel et al., 2018; Xu et al., 2018). sEVs have important roles in the protection of their contents, such as mRNAs, miRNAs, and proteins, from degradation, as well as in the delivery of their contents to recipient cells, which are needed for cell function (He et al., 2018; Kalluri and LeBleu, 2020). sEVs possess stem cell-like pro-regenerative properties, and the application of sEVs may prevent many of the adverse effects of stem cell transplantation therapy. More importantly, sEVs do not contain MHC-I or MHC-II proteins; as such, they overcome the disadvantages of stem cell transplantation therapy and seldom induce overt immune reactions (Roccaro et al., 2013). Previous studies have demonstrated that BMSC-derived sEVs (BMSC-sEVs) exhibit similar or identical therapeutic roles to those of BMSCs used in the treatment of bone defects, and miRNAs may induce osteoblast differentiation and bone formation (Tan et al., 2020; Wang and Thomsen, 2021). For example, Liu et al. reported that BMSC-sEVs expressing miR-130a can stimulate the PTEN/AKT signaling pathway during angiogenesis and bone remodeling (Liu et al., 2019), whereas Liao et al. demonstrated that BMSC-sEVs expressing miRNA-122-5p can promote osteoblast proliferation during osteonecrosis of the femoral head (Liao et al., 2019b). Taken together, these findings suggest that exploring the underlying mechanisms of bone regeneration induced by exosomal miRNAs may improve our understanding of osteogenesis and angiogenesis, thereby promoting the development of new treatment strategies.

Recently, hydrogels have generated considerable interest in the field of bone repair, as they involve a minimally invasive injection and form into a solid-like object in situ (Ingavle et al., 2019; Kocak et al., 2020). Polymer-based hydrogels have been used in tissue repair due to their structural uniformity, biodegradability, high permeability, biocompatibility, improved mechanical strength, and application ease (Hoffman et al., 2013). Chitosan (CS), a type of endogenous polysaccharide, has been widely used due to its many properties, including antibacterial activity and exogenous biomineralization capability (Petit et al., 2020). Among chitosanbased hydrogels, chitosan (CS)/ $\beta$-glycerophosphate $(\beta-\mathrm{GP})$ hydrogels have received much interest because of their thermosensitive properties and injectability (Wasupalli and Verma, 2020). Thermosensitive hydrogels exist as liquids at room temperature but form gels at body temperature, that is, most thermally-responsive hydrogels are soluble below a specific temperature, which is known as the lower critical solution temperature (LCST), but they are insoluble above this temperature (Bhattarai et al., 2010).

To our knowledge, the effects of $s E V$-loaded CS/ $\beta$-GP hydrogels on angiogenesis, which is critical for bone regeneration, have not yet been investigated (Wang et al., 2020). Here, an effort was made to fabricate a novel type of injectable hydrogel system (sEV@CS/ $\beta$-GP composite) consisting of the CS/ $\beta$-GP hydrogel and sEVs that was capable of forming a gel in situ at body temperature. The sEVs were released in a sustained and controlled manner, and the hydrogel was gradually degraded and internalized by human umbilical vein endothelial cells (HUVECs) and human BMSCs. Furthermore, the absorbed sEVs exhibited osteogenic and angiogenic properties. Previous studies have also reported that angiogenic activity was also promoted by miR-21 overexpression in BMSC-sEVs (Wu et al., 2020; Li G.-Q. et al., 2021; Zhang et al., 2021). In summary, sEV@CS/ $\beta-G P$ hydrogels can promote bone regeneration, which was likely mediated by miR-21 overexpression. These findings provide new insights on a promising therapeutic strategy for cell-free bone repair.

\section{MATERIALS AND METHODS}

\section{Cell Culture}

BMSCs, HUVECs, and HEK-293 cells were obtained from the cell bank of the Chinese Academy of Medical Sciences (Beijing, China). BMSCs were cultured in basal media (Cyagen Biosciences, Santa Clara, CA, United States), and HUVECs and HEK-293 cells were cultured in high-glucose Dulbecco's modified Eagle's medium (DMEM, Gibco BRL, Grand Island, NY, United States) supplemented with $10 \%$ fetal bovine serum (FBS) and $1 \%$ penicillin-streptomycin.

\section{Isolation and Characterization of SEVs sEV Isolation and Purification}

sEVs were isolated and purified from BMSC supernatants by ultracentrifugation as previously described (Colombo et al., 2014). Before isolation, BMSCs were incubated for $48 \mathrm{~h}$ in medium supplemented with 10\% sEV-depleted FBS (Umibio, Shanghai, China). The supernatant was collected and centrifuged at $300 \times \mathrm{g}$ for $10 \mathrm{~min}, 2000 \times \mathrm{g}$ for $20 \mathrm{~min}$, and $10,000 \times \mathrm{g}$ for $30 \mathrm{~min}$ to discard the cell debris, and centrifuged at $100,000 \times \mathrm{g}$ for $70 \mathrm{~min}$ to collect the sEVs. The pelleted sEVs were washed two 
times with PBS and centrifuged at $110,000 \times \mathrm{g}$ for $70 \mathrm{~min}$ to remove the contaminating proteins. All procedures were performed at $4^{\circ} \mathrm{C}$, and the sEVs were resuspended in PBS.

\section{sEV Identification and Internalization}

The size distribution of sEVs was determined by nanoparticle tracking analysis (NTA) with the NanoSight NS500 system (Malvern Instruments, Malvern, United Kingdom), and the morphology of sEVs was observed by transmission electron microscopy (TEM, Hitachi, Tokyo, Japan) as previously described (Wu et al., 2021). Western blotting was used to detect the expression of $\mathrm{sEV}$-specific surface markers, including CD63, CD81, and TSG101.

The uptake of sEVs by BMSCs and HUVECs was examined by labeling sEVs with the fluorescent dye PKH26 or PKH67 (SigmaAldrich, Darmstadt, Germany), according to the manufacturer's instructions, which were then incubated with BMSCs or HUVECs at $37^{\circ} \mathrm{C}$ for $24 \mathrm{~h}$. Subsequently, the cells were fixed with $4 \%$ paraformaldehyde, stained with DAPI for $10 \mathrm{~min}$, and observed by confocal microscopy (Nikon, Tokyo, Japan).

\section{Impact of sEVs on Osteogenic Differentiation}

\section{Alizarin Red Staining (ARS) and Alkaline Phosphatase (ALP) Activity}

The osteogenic differentiation of BMSCs was carried out $24 \mathrm{~h}$ after the incubation of sEVs or transfection of miRNA mimic or inhibitor. Briefly, the medium was replaced with osteogenic differentiation medium (Cyagen Biosciences), which was refreshed every $72 \mathrm{~h}$, supplemented with PBS $(200 \mu \mathrm{L})$ or different concentrations of BMSC-sEVs (50 or $100 \mu \mathrm{g} / \mathrm{ml}$ ). To assess mineralization, ARS was performed on day 14 after osteoinduction. Cells were stained with $2 \%$ ARS solution (Sigma-Aldrich) for $10 \mathrm{~min}$ and then washed with distilled water. To quantitatively determine matrix calcification, the cells were de-stained with $10 \%$ cetylpyridinium chloride in $10 \mathrm{mM}$ sodium phosphate for $30 \mathrm{~min}$, and the absorbance was measured at $562 \mathrm{~nm}$.

To assess ALP activity, the ALP assay kit (Beyotime, Jiangsu, China) was used. BMSCs were cultured in osteogenic differentiation medium and lysed on days 7 and 14 with $0.1 \%$ Triton $\mathrm{X}-100$ in Tris- $\mathrm{HCl}$ for $2 \mathrm{~h}$ at $4^{\circ} \mathrm{C}$. $p$-Nitrophenyl phosphate was added to the cell lysates, and the samples were incubated at $37^{\circ} \mathrm{C}$ for $15 \mathrm{~min}$. The normalized ALP activity was obtained by determining the total intracellular protein concentration with the Pierce BCA Protein Assay kit (Thermo Fisher Scientific, Waltham, MA, United States).

\section{Impact of sEVs on Angiogenesis Cell Proliferation Assay}

Three groups were prepared according to the BMSC-sEVs concentration $(0,50$, and $100 \mu \mathrm{g} / \mathrm{ml})$, with each group consisting of quadruplicate wells. HUVECs $\left(5 \times 10^{3}\right.$ cells/well $)$ were inoculated into 96-well plates and treated with the different BMSC-sEVs concentrations. The medium was changed every 2 days. To assess cell viability, the Cell Counting kit-8 (CCK-8;
Dojindo, Tokyo, Japan) was used. On days 1, 3, 5, and 7, the absorbance was measured at $450 \mathrm{~nm}$, and the growth curve was generated. CCK8 assay was also performed to test the promoting effect of gradient concentrations $(400,200,100,50,25$, and $0 \mu \mathrm{g} /$ $\mathrm{ml})$ of sEVs on cell proliferation.

\section{Cell Migration Assay}

HUVECs $\left(1 \times 10^{4}\right.$ cells/well $)$ were resuspended in serum-free medium and seeded into the upper chamber of Corning $8-\mu \mathrm{m}$ pore size transwell units (Corning, NY, United States) that were housed in 24-well plates. The lower chamber was filled with DMEM supplemented with $10 \% \mathrm{sEV}$-depleted FBS that was preincubated with PBS $(200 \mu \mathrm{L})$, BMSC-sEVs $(50 \mu \mathrm{g} / \mathrm{ml})$, or BMSCsEVs $(100 \mu \mathrm{g} / \mathrm{ml})$. The plates were incubated at $37^{\circ} \mathrm{C}$ for $24 \mathrm{~h}$. Thereafter, the cells attached to the upper surface of the filter membranes were removed, and those attached to the lower surface were stained with $0.1 \%$ crystal violet. Cell migration was observed by light microscopy (Leica, Solms, Germany).

\section{Tube Formation Assay}

The in vitro angiogenesis assay was conducted using Matrigel basement membrane matrix (BD Biosciences, San Jose, CA, United States), according to the manufacturer's instructions. Briefly, Matrigel was thawed overnight at $4^{\circ} \mathrm{C}$ and added to 96-well plates $(50 \mu \mathrm{L} /$ well $)$, which were then incubated at $37^{\circ} \mathrm{C}$. Thereafter, HUVECs $\left(2 \times 10^{4}\right.$ cells/well $)$ were resuspended in complete medium supplemented with $10 \%$ sEV-depleted FBS pre-incubated with PBS $(10 \mu \mathrm{L})$, BMSC-sEVs $(50 \mu \mathrm{g} / \mathrm{ml})$, or BMSC-sEVs $(100 \mu \mathrm{g} / \mathrm{ml})$. The plates were incubated at $37^{\circ} \mathrm{C}$ for $6 \mathrm{~h}$, and tube formation was observed by inverted microscopy. Five fields from each well were randomly selected, and the total tube length was measured with ImageJ software (National Institutes of Health, Bethesda, MD, United States).

\section{Quantitative Real-Time PCR (RT-qPCR) Analysis}

TRIzol (Invitrogen, Carlsbad, CA, United States) was used to isolate total RNA, and the Revert Aid First-Strand cDNA Synthesis kit (Vazyme, Jiangsu, China) was used to reverse transcribe RNA, according to the manufacturer's instructions. The sEV RNA Purification kit (Umibio, Shanghai, China) was used to extract miRNA, and the SYBR Green microRNA Assay kit (Applied Biosystems, Foster City, CA, United States) was used to synthesize cDNA. Real-time PCR was performed with the ABI PRISM $7900 \mathrm{H}$ T system using the SYBR Green Master-Mix kit (Applied Biosystems). GAPDH and U6 were used to normalize mRNA and miRNA expression levels, respectively, and the $2^{-\Delta \Delta C t}$ method was used to quantify the relative expression levels. All primer sequences are listed in Table $\mathbf{1 .}$

\section{Western Blotting}

The total protein concentrations in cells and sEVs were determined using the BCA Protein Assay kit. Proteins were separated by $10 \%$ SDS-PAGE, transferred to PVDF membranes, and probed with the appropriate primary and secondary antibodies. Immunoreactive bands were visualized using enhanced chemiluminescence reagents (Thermo Fisher Scientific) and quantified using ImageJ software. Primary 
TABLE 1 | List of primers used.

Gene

\begin{tabular}{l} 
\\
\hline OCN \\
OPN \\
Runx2 \\
VEGF \\
bFGF \\
ANG-1 \\
GAPDH \\
U6
\end{tabular}

U6

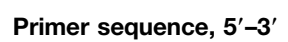

Forward

CACTCCTCGCCCTATTGGC

GAAGTITCGCAGACCTGACAT

TCAACGATCTGAGATITGTGGG

AGCGCCGAAGTCCAGAAAAC

AGAAGAGCGACCCTCACATCA

AGCGCCGAAGTCCAGAAAAC

CAGGGCTGCTITAACTCTGG

TGGAACGCTTCACGAATITGCG
Reverse

CCCTCCTGCTTGGACACAAAG

GTATGCACCATTCAACTCCTCG

GGGGAGGATाTTGAAGACGG

AGGGTCTCGATTGGATGGCA

CGGTTAGCACACACTCCTITG

TACTCTCACGACAGTTGCCAT

TGGGTGGAATCATATTGGAACA

GGAACGATACAGAGAAGATTAGC antibodies against CD63, CD81, TSG101, Calnexin, OCN, OPN, Runx2, VEGF, bFGF, ANG-1, and CD31 were obtained from Abcam (Cambridge, United Kingdom).

\section{Preparation and Characterization of Hydrogels \\ Hydrogel Preparation}

Thermosensitive CS/ $\beta$-GP hydrogels were prepared as previously described (Wu et al., 2019). Briefly, $0.2 \mathrm{~g}$ of CS (degree of deacetylation, 95\%; Sigma-Aldrich) was added to an acetic acid solution $(0.1 \mathrm{~mol} / \mathrm{L}, 10 \mathrm{ml})$ to obtain the $2 \%(\mathrm{w} / \mathrm{v}) \mathrm{CS}$ solution, followed by the dropwise addition of $1 \mathrm{ml}$ of $56 \%$ (w/ v) $\beta$-GP solution (Merck, Darmstadt, Germany) while stirring.

\section{Thermosensitivity}

The sol-to-gel transition behavior of the hydrogels at $37{ }^{\circ} \mathrm{C}$ was determined by the test tube inversion method (Park et al., 2012), which uses flow (sol) or no-flow (gel) criteria to assess flow when sample tubes are inverted for $30 \mathrm{~s}$ at a controlled temperature. The gelation time was determined by inverting the vial every $30 \mathrm{~s}$. Five independent duplicate tests were performed. The gelation time was recorded, and the average value was used.

\section{Scanning Electron Microscopy (SEM)}

Hydrogels were frozen at $-80^{\circ} \mathrm{C}$ for $24 \mathrm{~h}$ and lyophilized at $-40^{\circ} \mathrm{C}$ for $48 \mathrm{~h}$ in a freeze-dryer. Thereafter, the samples were cut with a sharp blade to obtain cross-sections and sputter-coated with a gold-platinum layer. Cross-sections were observed by SEM (XL30, PHILIPS, Eindhoven, Netherlands). The pore size was analyzed by ImageJ software, and the average pore size was calculated based on 50 pores in five randomly selected areas per sample.

\section{Viscosity}

A rheometer (Kinexus Ultra, Malvern, United Kingdom) equipped with a parallel plate with a diameter of $20 \mathrm{~mm}$ and a gap distance of $0.5 \mathrm{~mm}$ was used to measure the rheological properties of the hydrogel solution. The variations in the storage $\left(G^{\prime}\right)$ and loss $\left(G^{\prime \prime}\right)$ moduli of the samples were measured under constant strain $(0.1 \%)$ and frequency $(1.0 \mathrm{~Hz})$. The hydrogel solution was added to the parallel plate using a temperature range of $25-45^{\circ} \mathrm{C}$ and a heating rate of $0.5^{\circ} \mathrm{C} / \mathrm{min}$.

\section{Swelling and Degradation Behaviors}

Hydrogels $(n=3)$ were dried, weighed $\left(W_{d}\right)$, and rehydrated in PBS for $24 \mathrm{~h}$ at $37^{\circ} \mathrm{C}$. Samples were removed from PBS and weighed again $\left(\mathrm{W}_{\mathrm{s}}\right)$ at different time points after blotting the surface with filter paper (Yuan et al., 2019). The swelling ratio was calculated as follows $\left(\mathrm{W}_{\mathrm{s}}-\mathrm{W}_{\mathrm{d}}\right) / \mathrm{W}_{\mathrm{d}} \times 100 \%$

The degradation of the hydrogels was reflected in the weight loss, which was also studied in $\mathrm{PBS}$ at $37^{\circ} \mathrm{C}$. At given time points (days 1, 4, 7, 10, 14, and 21) after blotting the surface with filter paper, the hydrogel weight was measured. The weight loss ratio was defined as follows $\left(\mathrm{W}_{0}-\mathrm{W}_{\mathrm{t}}\right) / \mathrm{W}_{0} \times 100 \%$, where $\mathrm{W}_{0}$ is the initial weight of the sample, and $\mathrm{W}_{\mathrm{t}}$ is the weight of the sample at a specific time point.

\section{Mechanical Properties Test}

The mechanical properties of the hydrogels were determined with a universal mechanical analyzer (Instron, Norwood, MA, United States). Photo-crosslinked cylindrical hydrogels $(\mathrm{n}=4)$ with a height of $5 \mathrm{~mm}$ and a diameter of $10 \mathrm{~mm}$ were placed on the lower plate at a speed of $1 \mathrm{~mm} / \mathrm{min}$, and the compressive force was recorded until the hydrogels were deformed by the upper plate.

\section{Ability of sEVs/Gel Composites to Release sEVs}

sEVs $\left(1 \times 10^{8}\right.$ particles $\left./ \mathrm{mL}\right)$ were added to the composited hydrogels, and the release of sEVs from hydrogels was measured using the BCA Protein Assay kit. Briefly, the hydrogels were immersed in PBS in 24-well plates. At specific time points, the liquid on the surface of the hydrogels was collected, and the PBS in the wells was replaced. sEVs release was quantified and expressed as a percentage. Data were presented as mean \pm SD of three replicates.

\section{In Vivo Animal Experiments Surgical Procedures}

All procedures were approved by the Animal Research Committee of Peking Union Medical College Hospital (XHDW-2020-040), and all surgical procedures were performed in a sterile environment. Rats were anesthetized with an intraperitoneal injection of pentobarbital sodium $(50 \mathrm{mg} / \mathrm{kg})$. Thereafter, a $1.0-1.5-\mathrm{cm}$ midline sagittal incision was made on the scalp, and the calvarium was exposed by blunt dissection. Two full-thickness critical-size calvarial defects with a 
diameter of $5 \mathrm{~mm}$ each were created with a drill with a sterile bit, and 18 male rats at 8 weeks-of-age were randomly allocated into three groups as follows: PBS (control), CS/ $\beta$-GP hydrogel, and $\mathrm{CS} / \beta-\mathrm{GP}$ hydrogel with $200 \mu \mathrm{g}$ sEVs (sEV@CS/ $\beta$-GP). Thereafter, the bone defects were closed and sutured with degradable silk thread. All rats were housed individually and provided food and water in a temperature-controlled environment with regular use of prophylactic antibiotics.

\section{Micro-CT Analysis}

Rats were euthanized at 12 weeks after surgery, and the skulls were explanted and fixed in $4 \%(\mathrm{w} / \mathrm{v})$ paraformaldehyde. The morphology of the skulls was assessed using micro-computed tomography (CT) to determine the bone volume. The percentage of new bone volume to tissue volume (BV/TV), bone mineral density (BMD), trabecular number (Tb.N), and trabecular thickness (Tb.Th) were determined using Mimics software (Materialise, Leuven, Belgium).

\section{Histological, Immunohistochemical, and Immunofluorescence Analysis}

Specimens were fixed with $4 \%(\mathrm{w} / \mathrm{v})$ paraformaldehyde solution, decalcified with $5 \%(\mathrm{w} / \mathrm{v})$ EDTA, dehydrated in a graded series of alcohol solutions, and embedded in paraffin. Thereafter, 5- $\mu \mathrm{m}-$ thick cross-sections from mid-defect cranial regions were stained with hematoxylin and eosin (H\&E) and observed by light microscopy. Masson's trichrome staining was used to evaluate the degree of collagen maturation.

For immunohistochemical analysis, the cross-sections were treated with antigen retrieval buffer and incubated with a CD31 primary antibody $(1: 100)$ at $4^{\circ} \mathrm{C}$ overnight. Thereafter, the crosssections were incubated with a secondary antibody (1:250) at room temperature, followed by staining with $\mathrm{DAB}$ and counterstaining with hematoxylin. CD31 immunofluorescence staining was performed to examine new capillary formation. Cross-sections were incubated a CD31 primary antibody (1: 100) at $4{ }^{\circ} \mathrm{C}$ overnight and a secondary antibody (1:250) at room temperature for $1 \mathrm{~h}$ in the dark.

\section{Exosomal miR-21-Mediated Angiogenesis by Targeting SPRY2 \\ Luciferase Reporter Assay}

The 3 'untranslated region (UTR) of wild-type (wt) and mutant (mut) SPRY2 was amplified by PCR and individually inserted into the pGL3 plasmid. HEK293 cells $\left(5 \times 10^{4}\right)$ were seeded in 48 well plates and co-transfected with the wt or mut luciferase reporter (100 ng) and miR-21 mimics $(20 \mathrm{nM})$ or negative control (NCs) as indicated. At $48 \mathrm{~h}$ after transfection, the relative luciferase activity was detected with the Bright-Glo luciferase Assay system (Promega, Madison, WI, United States).

\section{Cell Transfection}

To examine the function of miR-21, HUVECs were transfected with miR-21 mimic or inhibitor and the respective NC (RiboBio, Guangzhou, China) using Lipofectamine 3000 reagent (Invitrogen). For SPRY2 overexpression, HUVECs were transfected with SPRY2 cDNA (Genechem, Shanghai, China) using Lipofectamine 3000 reagent.

\section{Statistical Analysis}

All experiments were performed at least in triplicate. All the data are presented as the mean \pm standard deviation (SD). Multiple group comparisons were performed by two-way analysis of variance with Tukey's post hoc test. Statistical analysis was carried out with GraphPad Prism 7.0 (GraphPad Software, La Jolla, CA, United States), and statistical significance was taken at $\left.{ }^{*}\right) p<0.05$ and $\left(^{* *}\right) p<0.01$.

\section{RESULTS}

\section{Characterization of sEVs}

sEVs were successfully isolated from BMSCs, and BMSC-sEVs were characterized by NTA analysis, TEM analysis, and western blotting. NTA analysis revealed the size distribution of the sEVs to consist of a single bell-shaped curve with a peak at approximately $120.0 \mathrm{~nm}$, and the percentage of the sEVs with a diameter in the range of $30-150 \mathrm{~nm}$ was $>99 \%$ (Figure 1A). TEM images showed the sEVs to be round or cup-shaped with a diameter in the range of $100-150 \mathrm{~nm}$ (Figure 1B). Western blotting analysis (Figure 1C) showed that sEVs, and not BMSCs, expressed CD63, CD81, and TSG101 but not calnexin (negative control). Representative fluorescence microscopy images (Figure 1D) showed that sEVs were observed in the cytoplasm, which was indicative of their attachment and internalization by BMSCs and HUVECs. In addition, immunoreactive PKH26 (red) and PKH67 (green) were observed around the nucleus (blue).

\section{Pro-Osteogenic and Pro-angiogenic Effects of BMSC-sEVs in vitro}

The promoting proliferative effect was enhanced as the concentrations of sEVs increased, while this effect was significantly reduced or faded when $\mathrm{sEVs}$ were more than $200 \mu \mathrm{g} / \mathrm{ml}$ (Supplementary Figure S1). Compared with controls, the results of ARS showed that the different concentrations of sEVs could enhance mineral deposition by BMSCs on day 14, and the highest concentration of sEVs could induce matrix mineralization and calcified nodule formation (Figure 2A). In quantitative analysis, there was significantly more calcium accumulation in the group treated with sEVs at $100 \mu \mathrm{g} / \mathrm{ml}$ than that in the group treated with sEVs at $50 \mu \mathrm{g} / \mathrm{ml}$ or the untreated group ( $p<0.05$; Figure 2B). ALP activity, an indicator of early-stage osteogenic differentiation of BMSCs, was significantly higher in cells exposed to both concentrations than that in control cells on days 7 and 14 ( $p<0.05$; Figure 2C), and the effects were dose-dependent. The mRNA levels of OCN, OPN, and Runx2 gradually increased in cells exposed to sEVs from days $7-14$, and the protein levels of OCN, OPN, and RUNX2 significantly increased on day 14 (Figure 2D), indicating that BMSC-sEVs can upregulate the expression of osteogenic genes $(p<0.05)$. The most significant increases in mRNA expression 

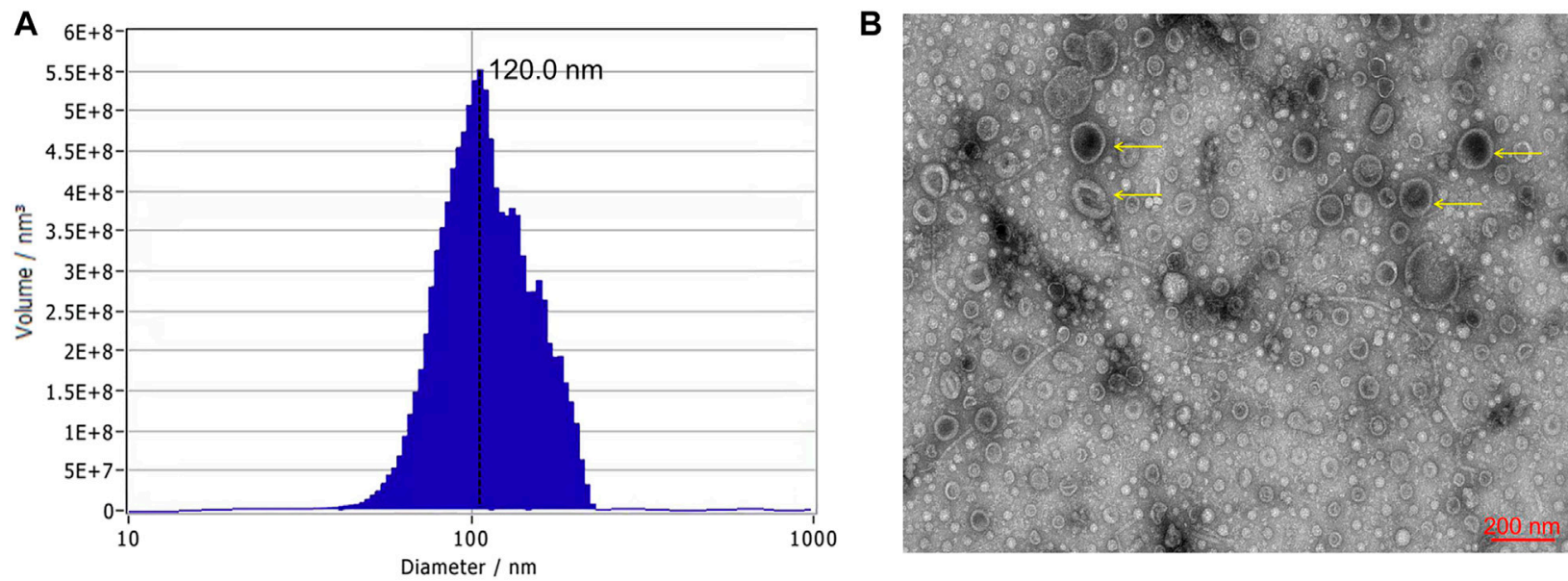

C

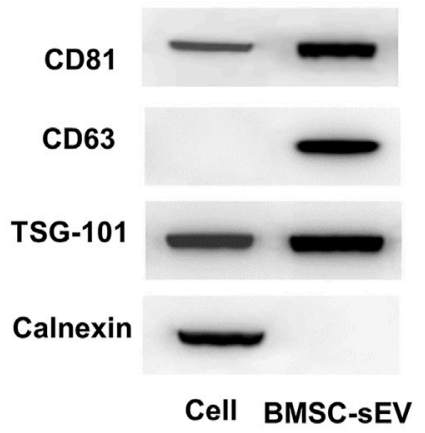

D

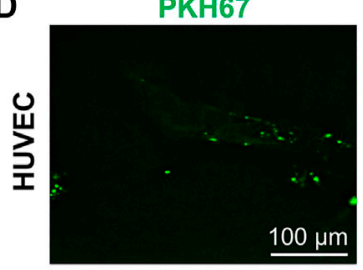

PKH26

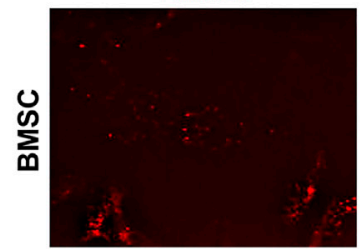

DAPI

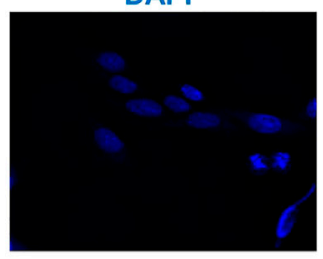

DAPI

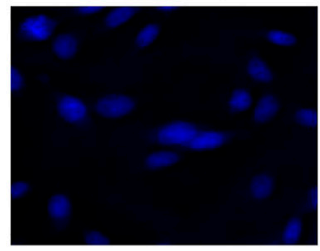

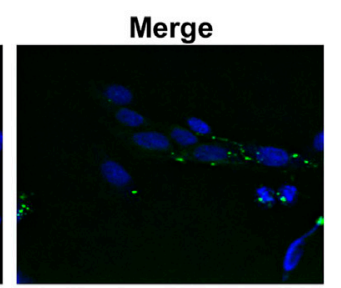

Merge

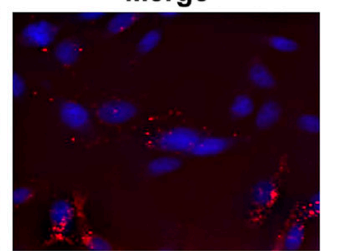

FIGURE 1 | Characterization and internalization of sEVs (A) Particle size distribution of sEVs detected by NTA and the mean diameter is 120.0 nm (B) Representative images of the morphology of sEVs by TEM. The yellow arrows indicate sEVs. Scale bar = $200 \mathrm{~nm}$ (C) Western blotting analysis of the exosomal proteins CD63, CD81, TSG101 and the negative marker Calnexin (D) Uptake of green fuorescent dye (PKH67)-labeled and red fuorescent dye (PKH26)-labeled sEVs by HUVECs and BMSCs, respectively.

were observed in cells treated with the highest concentration of sEVs (Figure 2E), revealing that the effects were dose-dependent.

As shown in Figures $\mathbf{3 A - D}$, the migratory capability of HUVECs exposed to sEVs for $24 \mathrm{~h}$ was greater than that in control cells, and the effects were dose-dependent. Furthermore, tube formation was increased in cells treated with sEVs at a concentration of $100 \mu \mathrm{g} / \mathrm{ml}$ compared with those treated at a concentration of $50 \mu \mathrm{g} / \mathrm{ml}$ or PBS. Compared with the control group, the proliferation of HUVECs exposed to BMSC-sEVs at both concentrations was also increased on days $1,3,5$, and 7 ( $p<$ $0.05)$, and the effects were dose-dependent (Figure 3E). Compared with controls, the protein levels of VEGF, bFGF, and ANG-1 increased in cells exposed to sEVs, with the highest concentration showing the most significant changes (Figure 3F). Likewise, compared with controls, the mRNA levels of VEGF, $b F G F$, and $A N G-1$ increased in cells exposed to sEVs on days 4-7, and the effects were dose-dependent (Figure 3G). These findings indicate that BMSC-sEVs can dose-dependently promote angiogenesis.

\section{Characterization of sEV Release From CS/ $\beta$-GP Hydrogels}

As shown in Figure 4A, the CS/ $\beta-\mathrm{GP}$ hydrogel was a colorless and transparent liquid at room temperature $\left(25^{\circ} \mathrm{C}\right)$. It underwent a sol-to-gel transition as the temperature increased, and at physiological temperature $\left(37^{\circ} \mathrm{C}\right)$, the $\mathrm{CS} / \beta-\mathrm{GP}$ hydrogel transformed into a non-flowing hydrogel. SEM results revealed that the hydrogel was porous and encased in a dense, thick polymeric wall (Figure 4B). Figure 4C shows the process by which hydrogels were loaded with sEVs. To verify the sol-to-gel transition, the viscosity was examined as a function of the temperature. The hydrogels had low elastic $\left(G^{\prime}\right)$ and viscous $\left(G^{\prime \prime}\right)$ moduli at $25^{\circ} \mathrm{C}$ and the intersection of $G^{\prime}$ and $G^{\prime \prime}$, which was characterized as $G^{\prime}<G^{\prime \prime}$ (Figure 4D), indicating their viscous nature, while the intersection of $G^{\prime}$ and $G^{\prime \prime}\left(G^{\prime}=G^{\prime \prime}\right)$ clearly revealed their gelation. Furthermore, the gap between the $G^{\prime}$ curve and the $G^{\prime \prime}$ curve for sEV-loaded hydrogels was slightly smaller compared with unloaded hydrogels. These results 

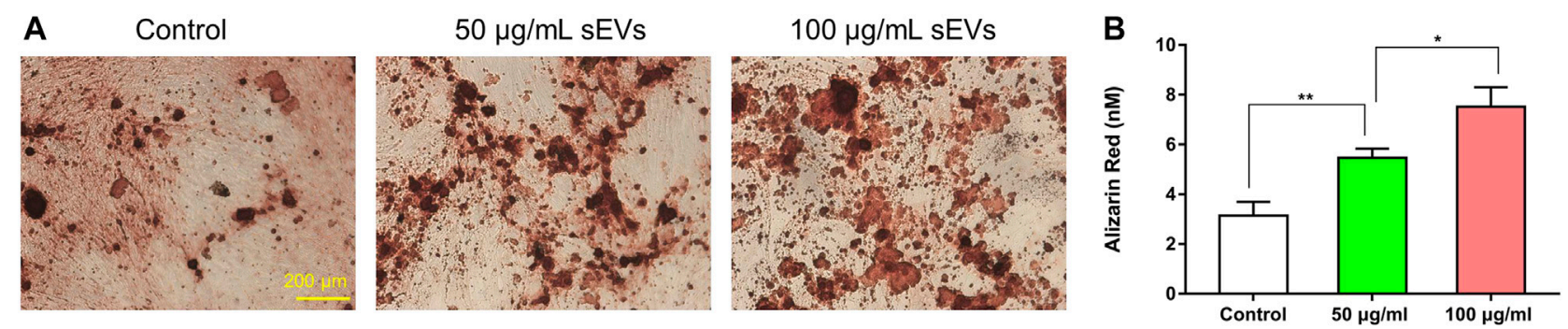

C

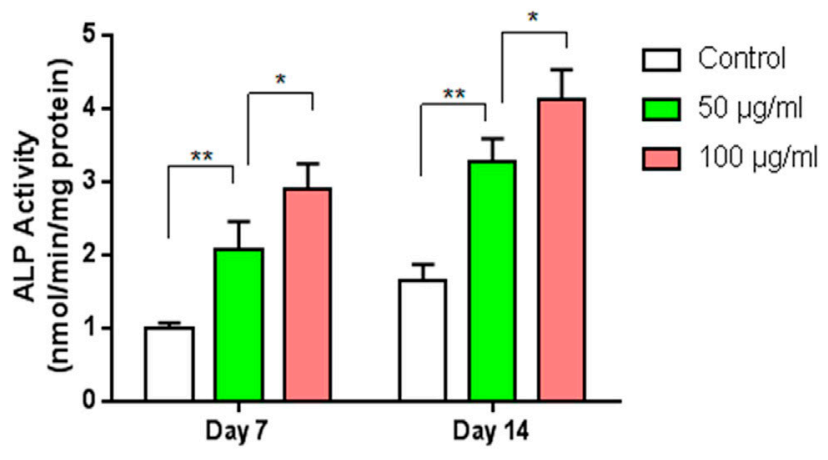

E

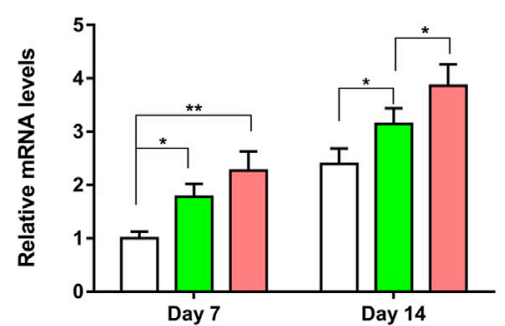

OPN

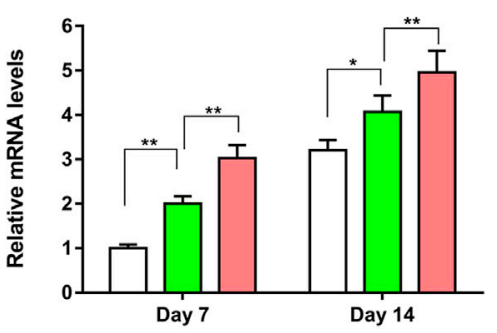

D

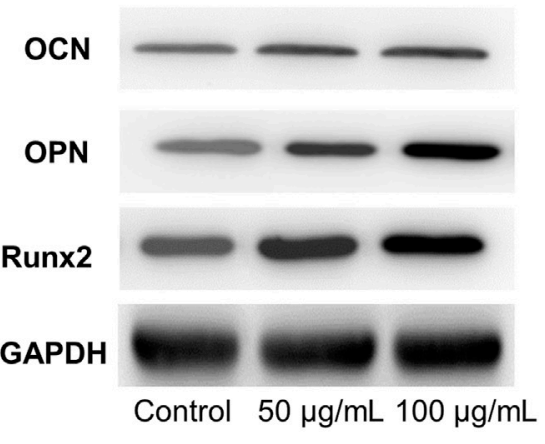

FIGURE 2 | Enhanced osteogenic effect of sEVs in vitro $\left(^{*}\right) p<0.05\left(^{\star \star}\right) p<0.01$ (A) ARS results from stimulation of sEVs on BMSCs after 14 days of culture. Scale bar $=200 \mu \mathrm{m}$ (B) Quantitative analysis of ARS (C) Quantifcation of ALP staining after incubation of BMSCs with sEVs for 7 and 14 days (D) Western blotting assay for the protein expression of OCN, OPN, and Runx2 (E) mRNA level of pro-osteogenic-related genes (OCN, OPN, and Runx2) in BMSCs exposed to different treatments.

indicate that the hydrogel behaved as a liquid before gelation and as a gel after gelation, that is, at physiological temperature and $\mathrm{pH}$.

Both CS/ $\beta$-GP and sEV@CS/ $\beta$-GP had good biodegradability and swelling behavior (Figure $4 \mathbf{E}$ ). The swelling ratio of the hydrogel increased with time, reaching equilibrium at approximately $6 \mathrm{~h}$, and there was no significant difference between the two hydrogels. Likewise, there was no difference in the weight loss of the two hydrogels, and the hydrolysis of imine bonds within the hydrogel in PBS was the likely mechanism. As shown in Figure 4F, there was a slight but non-significant increase in the gelation time when sEVs were added to $\mathrm{CS} / \beta-\mathrm{GP}$ hydrogels $(508 \pm 21$ and $569 \pm 26 \mathrm{~s})$. The maximal stress and strain of hydrogels were obtained from compressive curves, and the results indicated that both hydrogels had similar maximal stress and strain (Figure 4G). FTIR spectra revealed the addition of sEVs did not cause significant structural changes (Figure $\mathbf{4 H}$ ), as the sEVs may have interacted with certain functional groups to reduce the formation of hydrogen chemical bonds. A slight increase in wavelengths (from 4,254 to $4,268 \mathrm{~cm}^{-1}$ and 928 to $937 \mathrm{~cm}^{-1}$ ) indicated that sEVs had no impact on the thermosensitive hydrogels. Moreover, the sEV@CS/ $\beta-G P$ hydrogel showed good slow-release performance (Figure $4 \mathrm{I}$ ), with $80 \%$ of the sEVs releasing on day 8 and the release rate slowing after this time point.

\section{BMSC-sEVs Promote the Repair of Calvarial Defects in vivo}

The $3 \mathrm{D}$ reconstruction of micro-CT images of rat calvarial defects at 12 weeks are shown in Figure 5A. Compared with the control group, the formation of new bone, which filled the calvarial defects, was observed in CS/ $\beta-\mathrm{GP}$ and $s E V @ \mathrm{CS} / \beta-\mathrm{GP}$ groups, with the sEV@CS/ $\beta$-GP group showing a greater area of newly formed bone. The repair of these bone defects was further examined using quantitative approaches, and the BMD, BV/ $\mathrm{TV}$ ratio, and Tb.N in CS/ $\beta-\mathrm{GP}$ and sEV@CS/ $\beta-\mathrm{GP}$ groups 


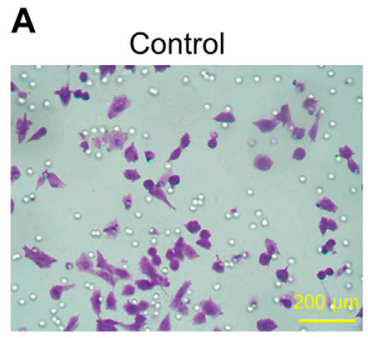

C Control
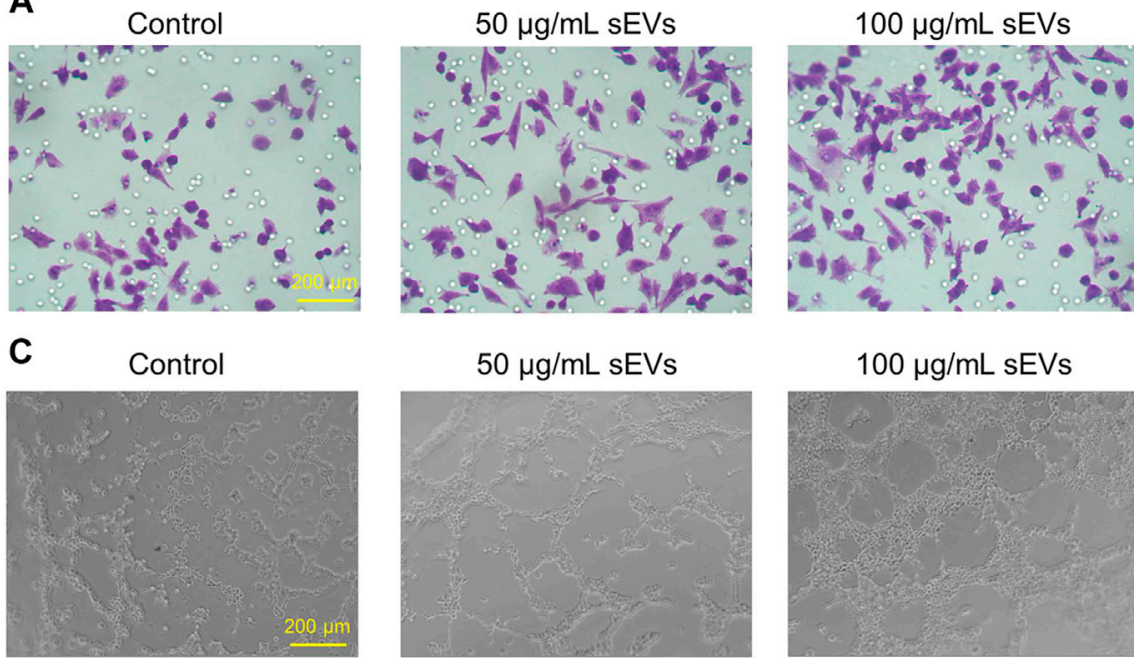

B

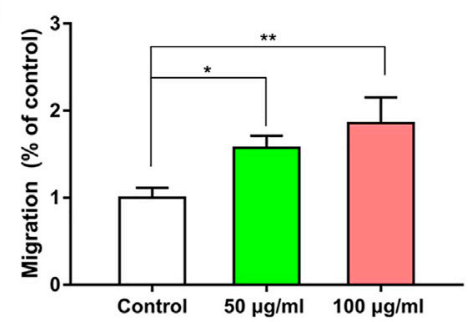

$50 \mu \mathrm{g} / \mathrm{mL}$ sEVs
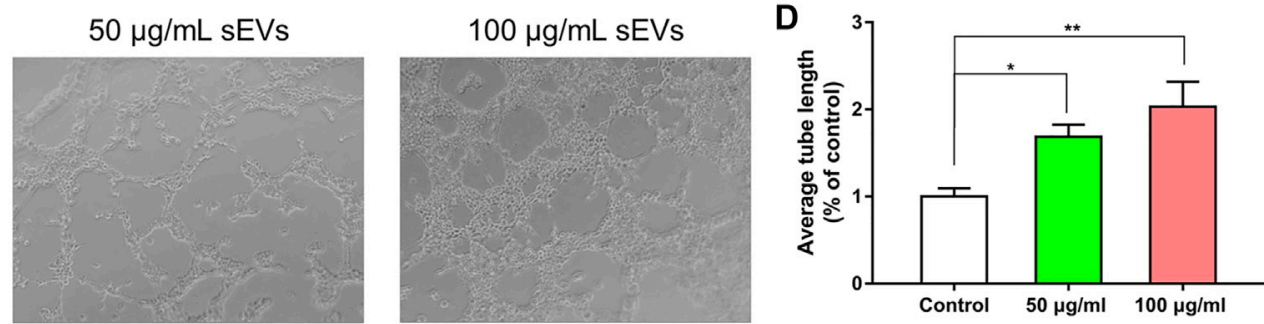

E
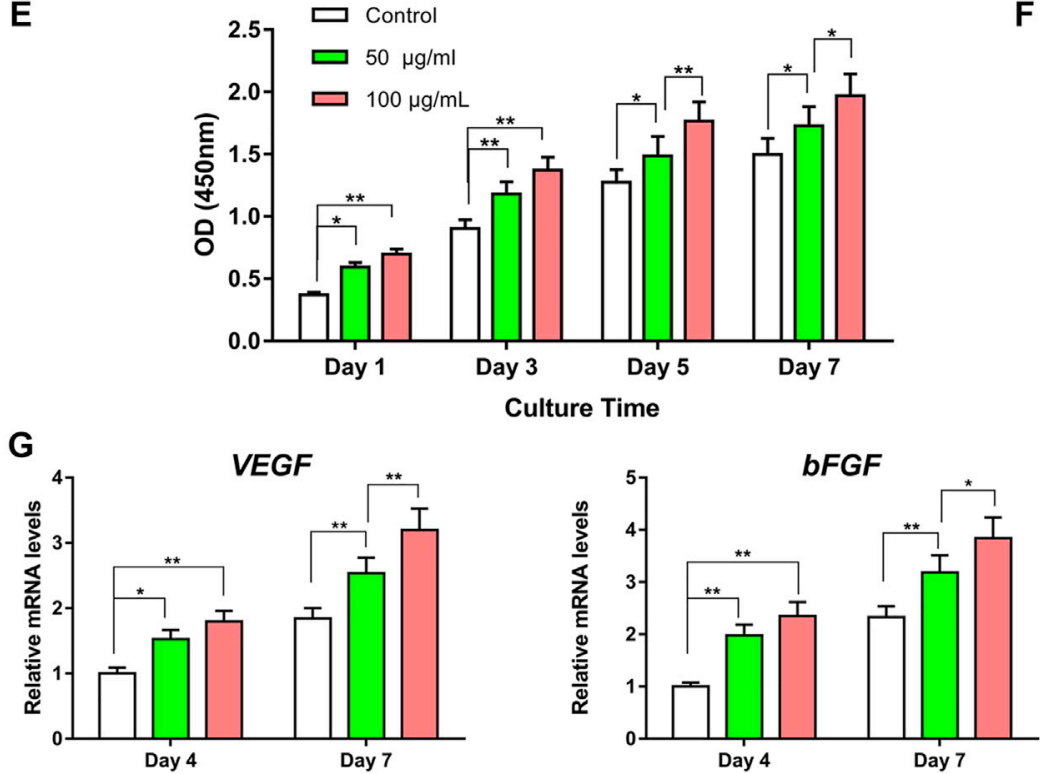

$\mathbf{F}$
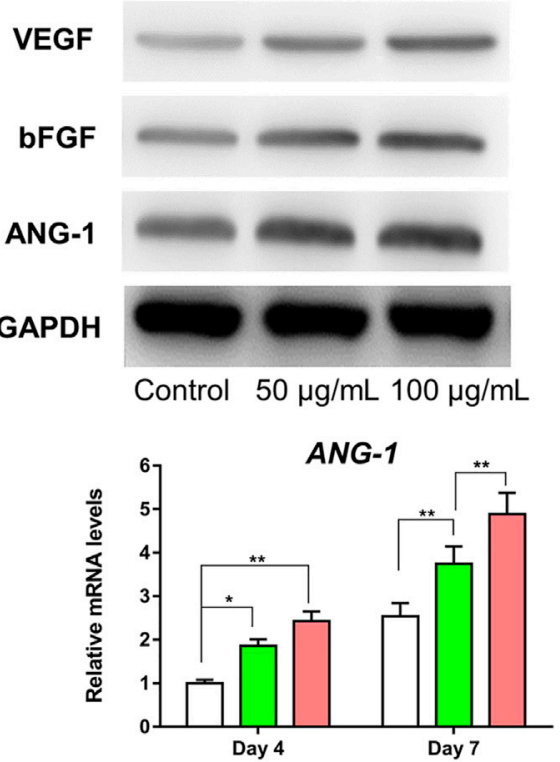

FIGURE 3 | Enhanced angiogenic effect of sEVs in vitro $\left(^{*}\right) p<0.05\left(^{* \star}\right) p<0.01$ (A) Transwell assay and quantitative analysis (B) of the cell migration rate (C) Tube formation by HUVECs and quantitative analysis (D) of the average tube length (E) CCK8 assay for the proliferation of HUVECs exposed to different treatments (F) Western blotting assay for the protein expression of VEGF, bFGF, and ANG-1 (G) mRNA level of pro-angiogenic-related genes (VEGF, bFGF, and ANG-1).

were all significantly higher than those in the control group, indicating that the release of sEVs from hydrogels and the thermosensitive property of hydrogels improved the bone healing capacity, whereas there was no difference in the $\mathrm{Tb}$.Th (Figure 5B). The results of histological staining indicated that the bone defects in the control group were mainly filled with fibrotic connective tissue, whereas newly formed bone was observed both along the border and in the center of the calvarial defects after application of CS/ $\beta$-GP hydrogels, with sEV@CS/ $\beta$-GP hydrogels showing a greater area of newly formed bone (Figures 5C,D, Supplementary Figure S2,3). These results were similar to those of micro-CT analysis. The results of CD31 immunohistochemical staining of right bone defect sections indicated that there were more CD31-positive cells in the sEV@CS/ $\beta$-GP group compared with the $C S / \beta-G P$ group, which was indicative of new vessel formation within the bone defect (Figure 5E), which was further verified by quantitative analysis (Figures 5F,G). These results reveal that $\mathrm{sEV}$ s promoted calvarial defect repair and enhanced angiogenesis and osteogenesis.

\section{BMSC-Derived Exosomal miR-21 Promotes Angiogenesis by Targeting SPRY2}

Previous studies have reported that miR-21 is expressed at a high level in sEVs derived from BMSCs (Lv et al., 2017). As such, we analyzed miRNA expression in BMSCs using an existing GEO dataset (GSE78865) and predicted the candidate target genes of miR-21 that contributed to 


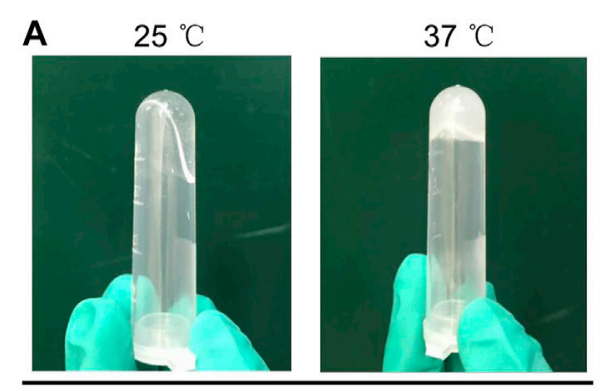

CS/ $\beta$-GP Hydrogel

C
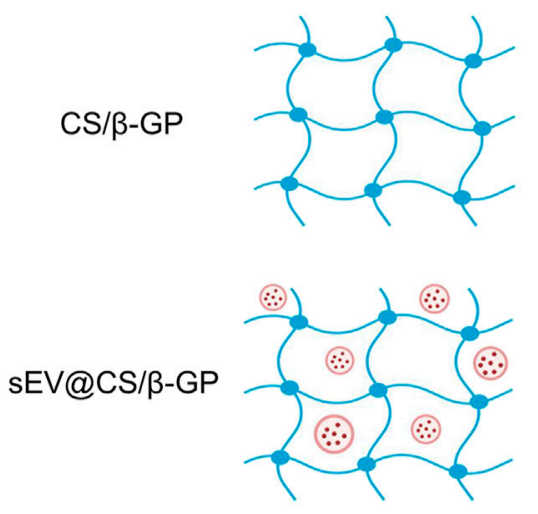

B

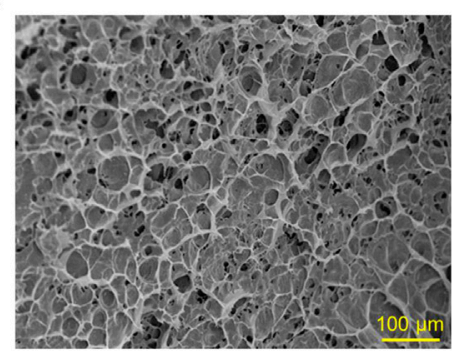

D
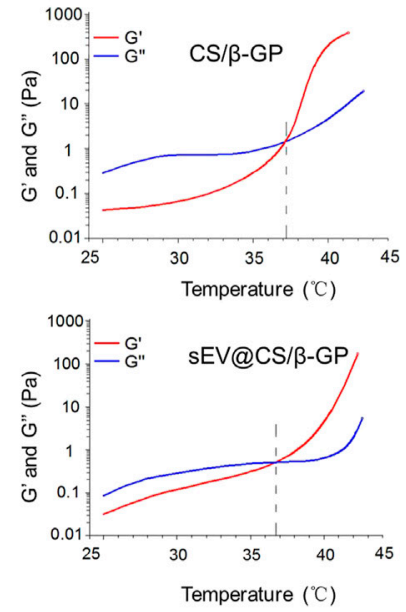

$\mathbf{H}$

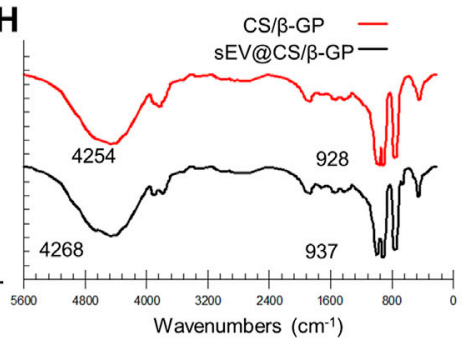

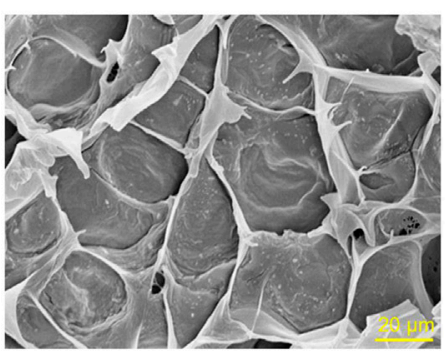

E $\left.{ }^{100}\right] \rightarrow \mathrm{CS} / \beta-\mathrm{GP}$
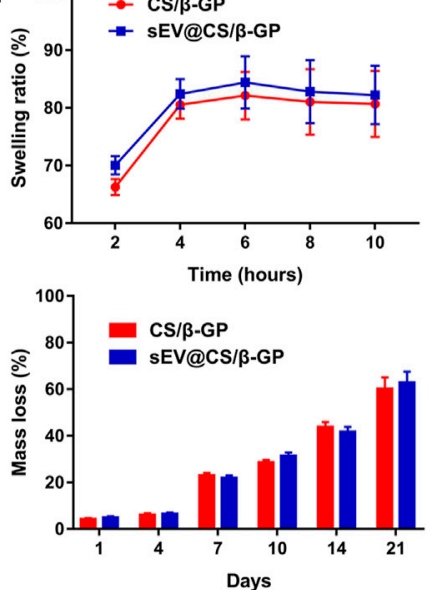

I

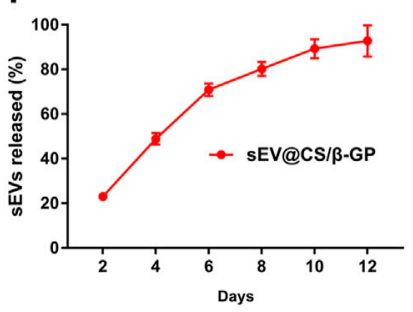

FIGURE 4 | Characterization and the sEV release ability of the CS/ $\beta$-GP hydrogel. ns: no significant difference (A) Sol-to-gel transition: a photograph of CS/ $\beta$-GP thermosensitive hydrogel at 25 and $37^{\circ} \mathrm{C}$ (B) SEM photomicrographs of the morphological structure of CS/ $\beta$-GP hydrogel (C) Schematic representation of the combination of sEVs and CS/ $\beta$-GP hydrogel (D) Typical temperature-dependent functions of $G^{\prime}$ and $G^{\prime \prime}$ for CS/ $\beta$-GP and sEV@CS/ $\beta$-GP hydrogels (E) Swelling rate and mass-remaining profiles of CS/ $\beta$-GP and sEV@CS/ $\beta$-GP hydrogels (F) Gelation time of CS/ $\beta$-GP and sEV@CS/ $\beta$-GP hydrogels (G) The compressive module of CS/ $\beta$-GP and sEV@CS/ $\beta$-GP hydrogels (H) FTIR (Fourier-transform infrared) spectra of CS/ $\beta$-GP and sEV@CS/ $\beta$-GP hydrogels (I) release profiles of sEVs from the sEV@CS/ $\beta$-GP hydrogel.

angiogenesis by exploring online databases, including TargetScan, miRanda and miRWalk. KEGG pathway enrichment analysis was performed for candidate target genes related to angiogenesis. To confirm the direct binding between miR-21 and the 3 '-UTR of its predicted target gene SPRY2, we performed reporter assays using a luciferase reporter plasmid containing the wt or mut SPRY2 3'-UTR with the miR-21 binding site (Figure 6A). Transfection of HUVECs with the miR-21 mimics could reduce luciferase activity compared to transfection with the control mimic, which was indicative of direct binding between miR-21 and the SPRY2 3-'UTR (Figure 6B).
To further explore the relationship between exosomal miR21 and SPRY2, rescue experiments were conducted. We transfected the miR-21 mimics or miR-NC into HUVECs, followed by co-transfection with a SPRY2-overexpressing plasmid (pcDNA-SPRY2). The results revealed that the migratory capacity of cells co-transfected with the miR-21 mimics and pcDNA-SPRY2 was enhanced compared with cells co-transfected with the miR-NC and pcDNA-SPRY2 (Figures 6C,D). The levels of angiogenic proteins in cells transfected with the miR-21 mimics were higher than those in control cells, and pcDNA-SPRY2 could abolish the effect of the miR21 mimics on angiogenesis (Figure 6E), indicating that 


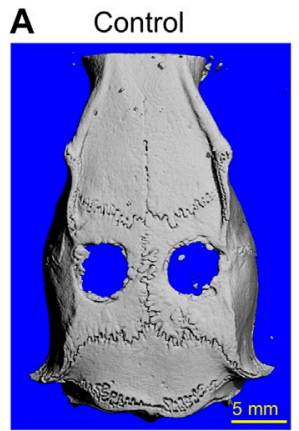

C Control

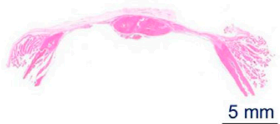

D Control

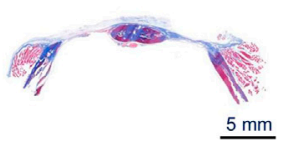

B

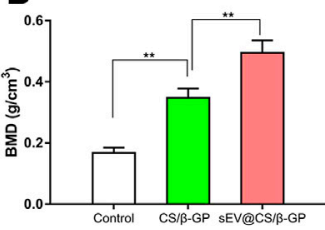

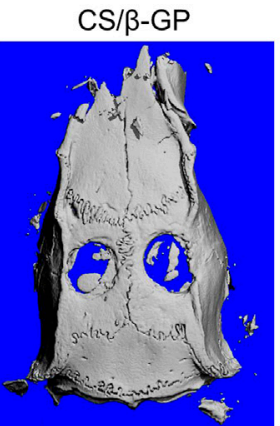

CS/B-GP

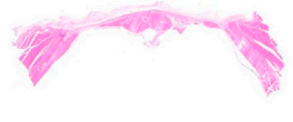

CS/ß-GP
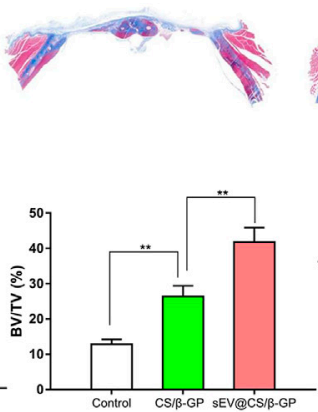

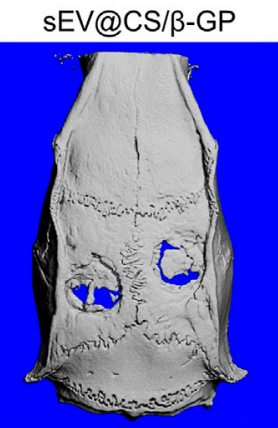

sEV@CS/ß-GP

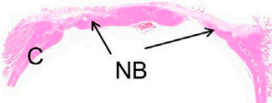

sEV@CS/ß-GP
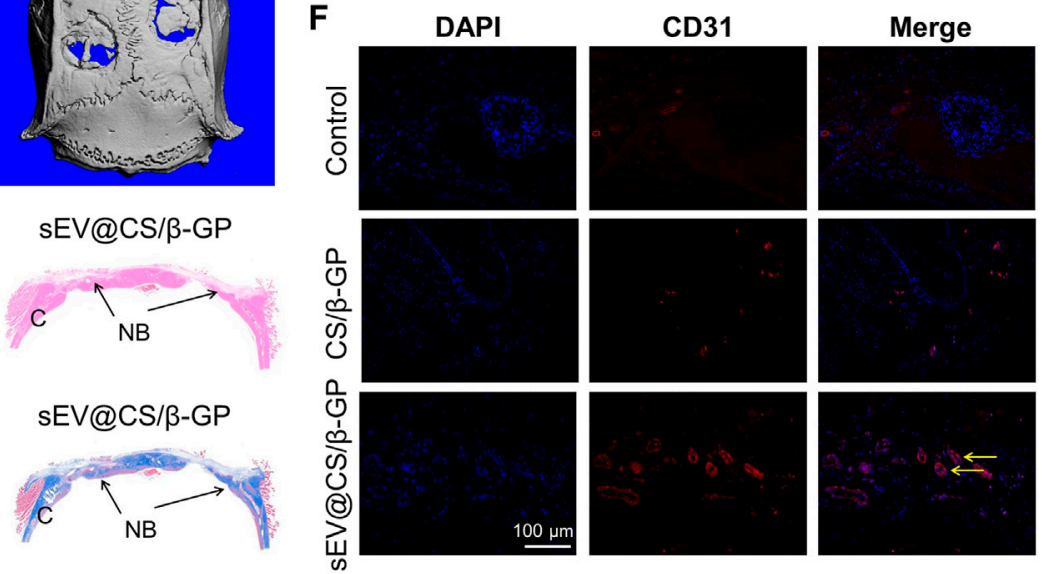

G
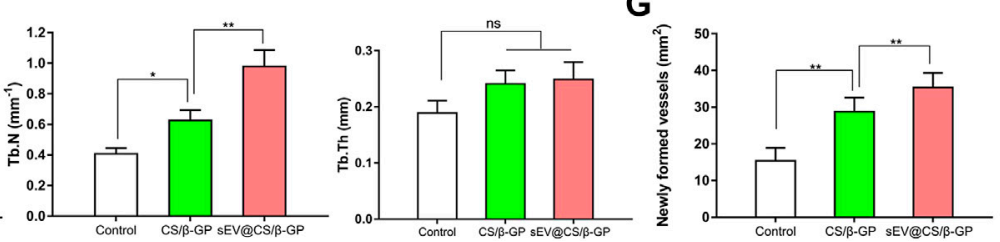

FIGURE 5 | sEV@CS/ $\beta$-GP hydrogel promoted bone formation in critical-sized rat calvarial defects $\left(^{\star}\right) p<0.05\left(^{\star \star}\right) p<0.01$, ns: no significant difference (A) MicroCT images of bone defects in each group after 12 weeks. Scale bar $=5 \mathrm{~mm}$ (B) Quantitative analysis of BMD, BV/TV ratio, Tb.N and Tb. Th in the diferent groups (C) H\&E staining and Masson's trichrome staining (D) in the three groups. C, cranium. NB, new bone (E) Immunohistochemical staining and immunofuorescence analysis (F) of the angiogenic marker CD31. Dark brown granules indicating positive staining are marked by black arrows, and yellow arrows mark the newly formed vessels (G) Quantitative analysis of newly formed vessels.

exosomal miR-21 can promote HUVEC migration and angiogenesis by targeting SPRY2.

\section{DISCUSSION}

Angiogenesis and osteogenesis are highly coupled processes that are indispensable for bone repair (Kusumbe et al., 2014). Large bone defects caused by trauma and certain diseases may not heal naturally and require regenerative scaffold implantation to promote tissue reconstruction (Dimitriou et al., 2011). Although a variety of bioengineering techniques that promote tissue regeneration with optimized materials are currently available, vascularization after scaffold implantation is still a major challenge (Potente et al., 2011; Rouwkema and Khademhosseini, 2016; Eelen et al., 2020). The osteogenic function of cells that promote bone regeneration requires a network of microvessels, which mediate the transport of circulating of cells, oxygen, nutrients, and waste products (Laschke and Menger, 2015). Besides, Zhao et al. modified the tetrahedral framework nucleic acid (tFNA) with aptamers to form aptamer-tFNA nanostructures, tFNA-Apt02 and tFNA-
AptVEGF, and they exhibited stronger angiogenesis, further provided a new and efficient proangiogenic approach (Zhao et al., 2021). Therefore, newly formed microvessels within grafts are critical for successful bone tissue engineering.

Mesenchymal stromal/stem cells (MSCs) have been widely applied because they can be obtained easily from adult tissues, as well as proliferate and differentiate into bone, adipose, or cartilage (Grayson et al., 2015). However, the direct use of MSCs for therapeutic purposes remains limited by many risk factors, such as tumor formation, thrombosis, and unwanted immune responses. Furthermore, the stimulation of localized healing by MSCs involves paracrine mechanisms (Gnecchi et al., 2005; Caplan and Correa, 2011), and the application of sEVs may overcome these limitations. Because of their ideal characteristics, sEVs are also favorable nanoscale drug carriers for the regeneration of tissues and the treatment of certain diseases (Liao et al., 2019a; Elsharkasy et al., 2020). For example, Dong et al. revealed that the use of fetal bovine serum-derived sEVs to carry Icariin could promote osteoblast proliferation and bone regeneration more effectively than Icariin alone (Dong et al., 2021). Wu et al. demonstrated that BMSC-derived sEVs and $\mathrm{Fe}_{3} \mathrm{O}_{4}$ nanoparticles under conditions of a static magnetic field 

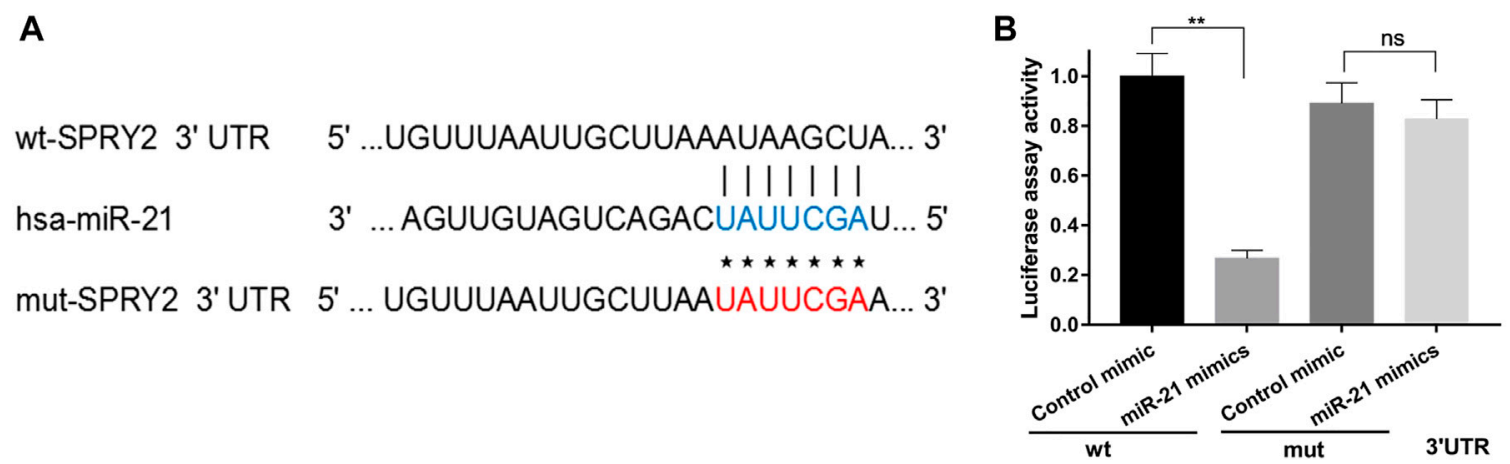

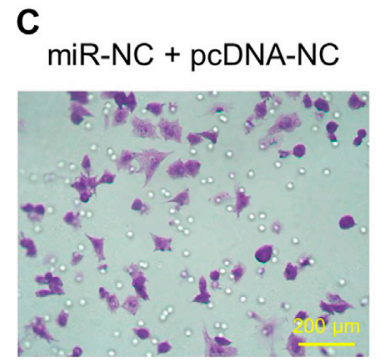

D
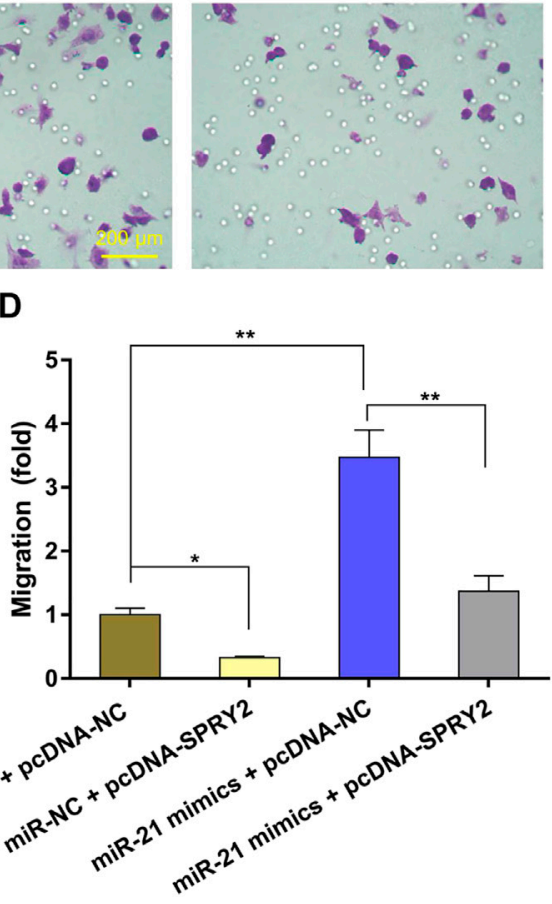

miR-21 mimics + pcDNA-NC

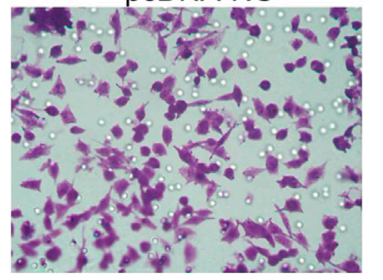

E miR-21 mimics

miR-NC PCDNA SPRY2 pcDNA-NC

VEGF
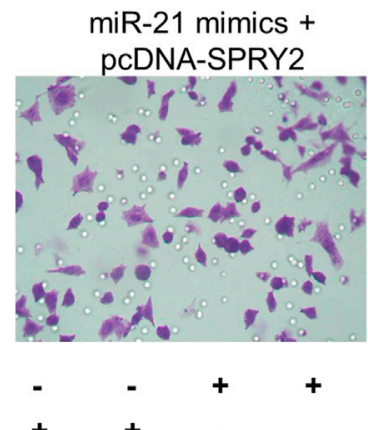

$+\quad+\quad-$
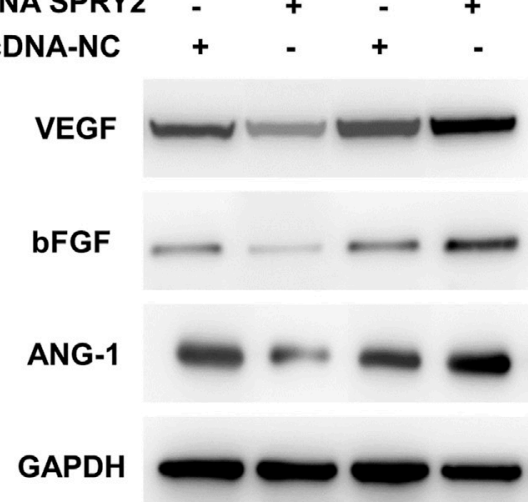

FIGURE 6 | Exosomal miR-21 promoted angiogenesis by targeting SPRY2 $\left(^{\star}\right) p<0.05\left(^{\star \star}\right) p<0.01$, ns: no significant difference. wt, wild-type; mut, mutant; NC, negative control (A) The miR-21 binding sequence in the $3^{\prime}$-UTR of SPRY2 (B) The miR-21 mimics transfection reduced luciferase activity when compared to control mimics transfection, which confirms that SPRY2 are the target genes of miR-21 (C) The transwell assay showing the cell migration when HUVECs were co-transfected with miR-21 mimics and pcDNA-SPRY2, and the quantitative analysis (D) indicated overexpression of SPRY2 suppressed the upregulation of the migration rate of HUVECs by miR-21 mimics (E) Western blotting assays showing that overexpression of SPRY2 prevented the upregulation of VEGF, bFGF, and ANG-1 protein expression by miR-21 mimics.

could facilitate bone regeneration and enhance wound healing (Wu et al., 2020; Wu et al., 2021). In the present study, we manufactured thermosensitive hydrogels loaded with BMSCderived $s E V s$ that could promote bone regeneration.

Hydrogels have been widely used in tissue bioengineering, drug delivery, and cell-based therapy. A recent study reported a biphasic hydrogel for osteochondral defect regeneration, which was fabricated via a thermally reactive, rapid cross-linking method (Liao et al., 2017). CS is an excellent excipient because it is non-toxic, stable, biodegradable, and sterilizable, which makes it a versatile material with application potential in biomedical and biotechnological fields (Kumar et al., 2004). CS can form a thermosensitive injectable hydrogel through crosslinking with $\beta$-GP via ionic interactions between the ammonium groups of CS and the phosphate groups of $\beta-\mathrm{GP}$, which can increase the gelation temperature and $\mathrm{pH}$ to the physiological range and restrict the immediate precipitation/deformation of the hydrogel (Bhattarai et al., 2010). The thermosetting of hydrogels has an added advantage, that is, once the homogeneous solution is injected into the tissue defect, the hydrogel forms and strengthens mechanically with time. In addition, the ability of polymer matrix hydrogels to expand and degrade makes them suitable vehicles for 
the encapsulation and delivery of numerous therapeutic agents, such as cells, growth factors, drugs, and proteins, to sites of tissue damage (Xu et al., 2019; Amiryaghoubi et al., 2020). These properties are ideal for sustained drug delivery applications, and CS/ $\beta$-GP hydrogels are attractive biomaterials because of their temperature sensitivity. The results of rheological experiments have revealed that the gelation time and temperature could be modulated by sEVs, as they slightly decreased the gelation time and temperature. As such, after $s E V$ loading, the resulting $s E V @ C S / \beta-G P$ hydrogels are attractive biomaterials for bone repair. Petit et al. reported that thermosensitive chitosan-based stain-loaded hydrogels decreased soft tissue inflammation and induced new bone formation (Petit et al., 2020), whereas Kocak et al. demonstrated that CS and hydroxyapatite composite materials loaded with low concentrations of heparin could stimulate angiogenesis and promote bone regeneration (Kocak et al., 2020).

MiRNAs regulate gene expression and biological functions by binding to the 3 '-UTR or amino acid coding sequence of target genes (Thomou et al., 2017). MiR-21, one of the most studied miRNAs, is involved in many biological processes (Kumarswamy et al., 2011). For example, Geng et al. reported that miR-21 can induce angiogenic differentiation of MSCs and promote blood vessel and bone formation (Geng et al., 2020). The role of SPRY2 in angiogenesis and the regulation of SPRY2 by the miR-23/27 cluster has been previously demonstrated (Zhou et al., 2011), as well as the regulation of SPRY2 by miR-21 (Thum et al., 2008). Li et al. fabricated the resultant bioswitchable nanocomposite by integrating the sticky-end tFNA (stFNA) and miRs (miR-21, miR-124, miR-335, and miR-2861), further promoting bone regeneration via inhibiting the expression of HDAC5 (Li S. et al., 2021). A similar research reported that exosomal miR21 derived from umbilical MSC-sEVs promoted angiogenesis by upregulating the NOTCH1/DLL4 pathway (Zhang et al., 2021). In this study, the results of luciferase assays revealed that SPRY2 expression was increased by the miR-21 mimics. Furthermore, SPRY2 significantly inhibited HUVEC migration and proliferation and mediated the negative feedback of major growth factors such as VEGF, bFGF, and ANG-1. The results of gain-of-function and loss-of-function assays indicated that SPRY2 overexpression in HUVECs could attenuate, but not entirely abolish, the effects of the miR-21 mimics on angiogenesis.

\section{CONCLUSION}

In summary, we developed an injectable homogeneous CS/ $\beta$-GP hydrogel solution, with gelation occurring at body temperature. The hydrogel had desirable structural and physical properties that promoted bone healing, and it served as a scaffold for sEVs. The sEV-loaded hydrogel could effectively promote bone healing in a

\section{REFERENCES}

Amiryaghoubi, N., Noroozi Pesyan, N., Fathi, M., and Omidi, Y. (2020). Injectable Thermosensitive Hybrid Hydrogel Containing Graphene Oxide and Chitosan rat model by enhancing angiogenesis, which may have been mediated by the upregulation of miR-21 expression in sEVs and the regulation of SPRY2 by miR-21. This study provides a new strategy for repairing bone defects with multifunctional controlled-sEV-release hydrogels, and this sEV-based therapy shows great potential in the future.

\section{DATA AVAILABILITY STATEMENT}

The original contributions presented in the study are included in the article/Supplementary Material, further inquiries can be directed to the corresponding authors.

\section{ETHICS STATEMENT}

The animal study was reviewed and approved by Animal Research Committee of Peking Union Medical College Hospital (XHDW-2020-040), Beijing, China.

\section{AUTHOR CONTRIBUTIONS}

DW, HQ and LL conducted most of the experimental work performance and drafted the manuscript. ZW, MY, ZL, and $\mathrm{HP}$ participated in data collection and related analysis. XW revised the manuscript. LL, CZ, and XW designed and supervised this project. All authors read and approved the final manuscript.

\section{FUNDING}

This work was supported by the National Key Research and Development Project of China (Grant No. 2018YFC1106303); Innovation Project of Peking Union Medical College Graduate Student (20191002-11).

\section{ACKNOWLEDGMENTS}

We are grateful to all the laboratory members for their technical advice and helpful discussion.

\section{SUPPLEMENTARY MATERIAL}

The Supplementary Material for this article can be found online at: https://www.frontiersin.org/articles/10.3389/fbioe.2021.829136/ full\#supplementary-material

as Dental Pulp Stem Cells Scaffold for Bone Tissue Engineering. Int. J. Biol. Macromolecules 162, 1338-1357. doi:10.1016/j.ijbiomac.2020.06.138

Bhattarai, N., Gunn, J., and Zhang, M. (2010). Chitosan-based Hydrogels for Controlled, Localized Drug Delivery. Adv. Drug Deliv. Rev. 62 (1), 83-99. doi:10.1016/j.addr.2009.07.019 
Bose, S., Roy, M., and Bandyopadhyay, A. (2012). Recent Advances in Bone Tissue Engineering Scaffolds. Trends Biotechnol. 30 (10), 546-554. doi:10.1016/ j.tibtech.2012.07.005

Caplan, A. I., and Correa, D. (2011). The MSC: an Injury Drugstore. Cell Stem Cell 9 (1), 11-15. doi:10.1016/j.stem.2011.06.008

Colombo, M., Raposo, G., and Théry, C. (2014). Biogenesis, Secretion, and Intercellular Interactions of Exosomes and Other Extracellular Vesicles. Annu. Rev. Cell Dev. Biol. 30, 255-289. doi:10.1146/annurev-cellbio-101512122326

Crane, G. M., Ishaug, S. L., and Mikos, A. G. (1995). Bone Tissue Engineering. Nat. Med. 1 (12), 1322-1324. doi:10.1038/nm1295-1322

Dimitriou, R., Jones, E., McGonagle, D., and Giannoudis, P. V. (2011). Bone Regeneration: Current Concepts and Future Directions. BMC Med. 9, 66. doi:10.1186/1741-7015-9-66

Dong, M., Wu, S., Xu, H., Yu, X., Wang, L., Bai, H., et al. (2021). FBS-derived Exosomes as a Natural Nano-Scale Carrier for Icariin Promote Osteoblast Proliferation. Front. Bioeng. Biotechnol. 9, 615920. doi:10.3389/ fbioe.2021.615920

Eelen, G., Treps, L., Li, X., and Carmeliet, P. (2020). Basic and Therapeutic Aspects of Angiogenesis Updated. Circ. Res. 127 (2), 310-329. doi:10.1161/ CIRCRESAHA.120.316851

Elsharkasy, O. M., Nordin, J. Z., Hagey, D. W., de Jong, O. G., Schiffelers, R. M., Andaloussi, S. E., et al. (2020). Extracellular Vesicles as Drug Delivery Systems: Why and How? Adv. Drug Deliv. Rev. 159, 332-343. doi:10.1016/ j.addr.2020.04.004

Fu, X., Liu, G., Halim, A., Ju, Y., Luo, Q., and Song, A. G. (2019). Mesenchymal Stem Cell Migration and Tissue Repair. Cells 8 (8), 784. doi:10.3390/ cells 8080784

Geng, Z., Yu, Y., Li, Z., Ma, L., Zhu, S., Liang, Y., et al. (2020). miR-21 Promotes Osseointegration and Mineralization through Enhancing Both Osteogenic and Osteoclastic Expression. Mater. Sci. Eng. C 111, 110785. doi:10.1016/ j.msec.2020.110785

Gnecchi, M., He, H., Liang, O. D., Melo, L. G., Morello, F., Mu, H., et al. (2005). Paracrine Action Accounts for Marked protection of Ischemic Heart by AktModified Mesenchymal Stem Cells. Nat. Med. 11 (4), 367-368. doi:10.1038/ nm0405-367

Grayson, W. L., Bunnell, B. A., Martin, E., Frazier, T., Hung, B. P., and Gimble, J. M. (2015). Stromal Cells and Stem Cells in Clinical Bone Regeneration. Nat. Rev. Endocrinol. 11 (3), 140-150. doi:10.1038/nrendo.2014.234

He, C., Zheng, S., Luo, Y., and Wang, B. (2018). Exosome Theranostics: Biology and Translational Medicine. Theranostics 8 (1), 237-255. doi:10.7150/thno.21945

Hoffman, M. D., Xie, C., Zhang, X., and Benoit, D. S. W. (2013). The Effect of Mesenchymal Stem Cells Delivered via Hydrogel-Based Tissue Engineered Periosteum on Bone Allograft Healing. Biomaterials 34 (35), 8887-8898. doi:10.1016/j.biomaterials.2013.08.005

Huey, D. J., Hu, J. C., and Athanasiou, K. A. (2012). Unlike Bone, Cartilage Regeneration Remains Elusive. Science 338 (6109), 917-921. doi:10.1126/ science. 1222454

Ingavle, G. C., Gionet-Gonzales, M., Vorwald, C. E., Bohannon, L. K., Clark, K., Galuppo, L. D., et al. (2019). Injectable Mineralized Microsphere-Loaded Composite Hydrogels for Bone Repair in a Sheep Bone Defect Model. Biomaterials 197, 119-128. doi:10.1016/j.biomaterials.2019.01.005

Kalluri, R., and LeBleu, V. S. (2020). The Biology , Function , and Biomedical Applications of Exosomes. Science 367 (6478). doi:10.1126/science.aau6977

Kawai, M., Mödder, U. I., Khosla, S., and Rosen, C. J. (2011). Emerging Therapeutic Opportunities for Skeletal Restoration. Nat. Rev. Drug Discov. 10 (2), 141-156. doi:10.1038/nrd3299

Khademhosseini, A., and Langer, R. (2016). A Decade of Progress in Tissue Engineering. Nat. Protoc. 11 (10), 1775-1781. doi:10.1038/nprot.2016.123

Kocak, F. Z., Talari, A. C. S., Yar, M., and Rehman, I. U. (2020). In-Situ Forming pH and Thermosensitive Injectable Hydrogels to Stimulate Angiogenesis: Potential Candidates for Fast Bone Regeneration Applications. Ijms 21 (5), 1633. doi:10.3390/ijms21051633

Kumar, M. N. V. R., Muzzarelli, R. A. A., Muzzarelli, C., Sashiwa, H., and Domb, A. J. (2004). Chitosan Chemistry and Pharmaceutical Perspectives. Chem. Rev. 104 (12), 6017-6084. doi:10.1021/cr030441b
Kumarswamy, R., Volkmann, I., and Thum, T. (2011). Regulation and Function of miRNA-21 in Health and Disease. RNA Biol. 8 (5), 706-713. doi:10.4161/ rna.8.5.16154

Kusumbe, A. P., Ramasamy, S. K., and Adams, R. H. (2014). Coupling of Angiogenesis and Osteogenesis by a Specific Vessel Subtype in Bone. Nature 507 (7492), 323-328. doi:10.1038/nature 13145

Langer, R., and Vacanti, J. P. (1993). Tissue Engineering. Science 260 (5110), 920-926. doi:10.1126/science.8493529

Laschke, M. W., and Menger, M. D. (2015). Adipose Tissue-Derived Microvascular Fragments: Natural Vascularization Units for Regenerative Medicine. Trends Biotechnol. 33 (8), 442-448. doi:10.1016/j.tibtech.2015.06.001

Li, G.-Q., Fang, Y.-X., Liu, Y., Meng, F.-R., Wu, X., Zhang, C.-W., et al. (2021a). MicroRNA-21 from Bone Marrow Mesenchymal Stem Cell-Derived Extracellular Vesicles Targets TET1 to Suppress KLF4 and Alleviate Rheumatoid Arthritis. Ther. Adv. Chronic Dis. 12, 204062232110073. doi:10.1177/20406223211007369

Li, S., Liu, Y., Tian, T., Zhang, T., Lin, S., Zhou, M., et al. (2021b). Bioswitchable Delivery of microRNA by Framework Nucleic Acids: Application to Bone Regeneration. Small 17 (47), 2104359. doi:10.1002/smll.202104359

Li, W., Liu, Y., Zhang, P., Tang, Y., Zhou, M., Jiang, W., et al. (2018). TissueEngineered Bone Immobilized with Human Adipose Stem Cells-Derived Exosomes Promotes Bone Regeneration. ACS Appl. Mater. Inter. 10 (6), 5240-5254. doi:10.1021/acsami.7b17620

Liao, J., Tian, T., Shi, S., Xie, X., Ma, Q., Li, G., et al. (2017). The Fabrication of Biomimetic Biphasic CAN-PAC Hydrogel with a Seamless Interfacial Layer Applied in Osteochondral Defect Repair. Bone Res. 5, 17018. doi:10.1038/ boneres. 2017.18

Liao, W., Du, Y., Zhang, C., Pan, F., Yao, Y., Zhang, T., et al. (2019a). Exosomes: The Next Generation of Endogenous Nanomaterials for Advanced Drug Delivery and Therapy. Acta Biomater. 86, 1-14. doi:10.1016/ j.actbio.2018.12.045

Liao, W., Ning, Y., Xu, H.-J., Zou, W.-Z., Hu, J., Liu, X.-Z., et al. (2019b). BMSCderived Exosomes Carrying microRNA-122-5p Promote Proliferation of Osteoblasts in Osteonecrosis of the Femoral Head. Clin. Sci. (Lond) 133 (18), 1955-1975. doi:10.1042/CS20181064

Liu, L., Liu, Y., Feng, C., Chang, J., Fu, R., Wu, T., et al. (2019). Lithium-containing Biomaterials Stimulate Bone Marrow Stromal Cell-Derived Exosomal miR130a Secretion to Promote Angiogenesis. Biomaterials 192, 523-536. doi:10.1016/j.biomaterials.2018.11.007

Lopes, D., Martins-Cruz, C., Oliveira, M. B., and Mano, J. F. (2018). Bone Physiology as Inspiration for Tissue Regenerative Therapies. Biomaterials 185, 240-275. doi:10.1016/j.biomaterials.2018.09.028

Lv, C., Yang, S., Chen, X., Zhu, X., Lin, W., Wang, L., et al. (2017). MicroRNA-21 Promotes Bone Mesenchymal Stem Cells Migration In Vitro by Activating PI3K/Akt/MMPs Pathway. J. Clin. Neurosci. 46, 156-162. doi:10.1016/ j.jocn.2017.07.040

Park, M. H., Joo, M. K., Choi, B. G., and Jeong, B. (2012). Biodegradable Thermogels. Acc. Chem. Res. 45 (3), 424-433. doi:10.1021/ar200162j

Petit, C., Batool, F., Stutz, C., Anton, N., Klymchenko, A., Vandamme, T., et al. (2020). Development of a Thermosensitive Statin Loaded Chitosan-Based Hydrogel Promoting Bone Healing. Int. J. Pharmaceutics 586, 119534. doi:10.1016/j.jpharm.2020.119534

Potente, M., Gerhardt, H., and Carmeliet, P. (2011). Basic and Therapeutic Aspects of Angiogenesis. Cell 146 (6), 873-887. doi:10.1016/j.cell.2011.08.039

Roccaro, A. M., Sacco, A., Maiso, P., Azab, A. K., Tai, Y.-T., Reagan, M., et al. (2013). BM Mesenchymal Stromal Cell-Derived Exosomes Facilitate Multiple Myeloma Progression. J. Clin. Invest. 123 (4), 1542-1555. doi:10.1172/JCI66517

Rouwkema, J., and Khademhosseini, A. (2016). Vascularization and Angiogenesis in Tissue Engineering: Beyond Creating Static Networks. Trends Biotechnol. 34 (9), 733-745. doi:10.1016/j.tibtech.2016.03.002

Sissung, T. M., and Figg, W. D. (2020). Stem Cell Clinics: Risk of Proliferation. Lancet Oncol. 21 (2), 205-206. doi:10.1016/S1470-2045(19)30787-9

Tan, S. H. S., Wong, J. R. Y., Sim, S. J. Y., Tjio, C. K. E., Wong, K. L., Chew, J. R. J., et al. (2020). Mesenchymal Stem Cell Exosomes in Bone Regenerative Strategies-A Systematic Review of Preclinical Studies. Mater. Today Bio 7, 100067. doi:10.1016/j.mtbio.2020.100067 
Thomou, T., Mori, M. A., Dreyfuss, J. M., Konishi, M., Sakaguchi, M., Wolfrum, C., et al. (2017). Adipose-derived Circulating miRNAs Regulate Gene Expression in Other Tissues. Nature 542 (7642), 450-455. doi:10.1038/nature21365

Thum, T., Gross, C., Fiedler, J., Fischer, T., Kissler, S., Bussen, M., et al. (2008). MicroRNA-21 Contributes to Myocardial Disease by Stimulating MAP Kinase Signalling in Fibroblasts. Nature 456 (7224), 980-984. doi:10.1038/nature07511

Tkach, M., and Théry, C. (2016). Communication by Extracellular Vesicles: Where We Are and where We Need to Go. Cell 164 (6), 1226-1232. doi:10.1016/ j.cell.2016.01.043

van Niel, G., D’Angelo, G., and Raposo, G. (2018). Shedding Light on the Cell Biology of Extracellular Vesicles. Nat. Rev. Mol. Cell Biol 19 (4), 213-228. doi:10.1038/nrm.2017.125

Wang, L., Wang, J., Zhou, X., Sun, J., Zhu, B., Duan, C., et al. (2020). A New SelfHealing Hydrogel Containing hucMSC-Derived Exosomes Promotes Bone Regeneration. Front. Bioeng. Biotechnol. 8, 564731. doi:10.3389/ fbioe.2020.564731

Wang, X., and Thomsen, P. (2021). Mesenchymal Stem Cell-Derived Small Extracellular Vesicles and Bone Regeneration. Basic Clin. Pharmacol. Toxicol. 128 (1), 18-36. doi:10.1111/bcpt.13478

Wasupalli, G. K., and Verma, D. (2020). Injectable and Thermosensitive Nanofibrous Hydrogel for Bone Tissue Engineering. Mater. Sci. Eng. C 107, 110343. doi:10.1016/j.msec.2019.110343

Wu, D., Chang, X., Tian, J., Kang, L., Wu, Y., Liu, J., et al. (2021). Bone Mesenchymal Stem Cells Stimulation by Magnetic Nanoparticles and a Static Magnetic Field: Release of Exosomal miR-1260a Improves Osteogenesis and Angiogenesis. J. Nanobiotechnol 19 (1), 209. doi:10.1186/ s12951-021-00958-6

Wu, D., Kang, L., Tian, J., Wu, Y., Liu, J., Li, Z., et al. (2020). Exosomes Derived from Bone Mesenchymal Stem Cells with the Stimulation of Fe3O4 Nanoparticles and Static Magnetic Field Enhance Wound Healing through Upregulated miR-21-5p. Ijn 15, 7979-7993. doi:10.2147/IJN.S275650

Wu, J., Zheng, K., Huang, X., Liu, J., Liu, H., Boccaccini, A. R., et al. (2019). Thermally Triggered Injectable Chitosan/silk Fibroin/bioactive Glass Nanoparticle Hydrogels for In-Situ Bone Formation in Rat Calvarial Bone Defects. Acta Biomater. 91, 60-71. doi:10.1016/j.actbio.2019.04.023

Xu, R., Rai, A., Chen, M., Suwakulsiri, W., Greening, D. W., and Simpson, R. J. (2018). Extracellular Vesicles in Cancer - Implications for Future Improvements in Cancer Care. Nat. Rev. Clin. Oncol. 15 (10), 617-638. doi:10.1038/s41571-018-0036-9

Xu, X., Gu, Z., Chen, X., Shi, C., Liu, C., Liu, M., et al. (2019). An Injectable and Thermosensitive Hydrogel: Promoting Periodontal Regeneration by
Controlled-Release of Aspirin and Erythropoietin. Acta Biomater. 86, 235-246. doi:10.1016/j.actbio.2019.01.001

Yuan, X., Amarnath Praphakar, R., Munusamy, M. A., Alarfaj, A. A., Suresh Kumar, S., and Rajan, M. (2019). Mucoadhesive Guargum Hydrogel Interconnected Chitosan-G-Polycaprolactone Micelles for Rifampicin Delivery. Carbohydr. Polym. 206, 1-10. doi:10.1016/j.carbpol.2018.10.098

Zaidi, M. (2007). Skeletal Remodeling in Health and Disease. Nat. Med. 13 (7), 791-801. doi:10.1038/nm1593

Zhang, B., Skelly, J. D., Maalouf, J. R., Ayers, D. C., and Song, J. (2019). Multifunctional Scaffolds for Facile Implantation, Spontaneous Fixation, and Accelerated Long Bone Regeneration in Rodents. Sci. Transl. Med. 11 (502). doi:10.1126/scitranslmed.aau7411

Zhang, Y., Xie, Y., Hao, Z., Zhou, P., Wang, P., Fang, S., et al. (2021). Umbilical Mesenchymal Stem Cell-Derived Exosome-Encapsulated Hydrogels Accelerate Bone Repair by Enhancing Angiogenesis. ACS Appl. Mater. Inter. 13 (16), 18472-18487. doi:10.1021/acsami.0c22671

Zhao, D., Liu, M., Li, J., Xiao, D., Peng, S., He, Q., et al. (2021). Angiogenic Aptamer-Modified Tetrahedral Framework Nucleic Acid Promotes Angiogenesis In Vitro and In Vivo. ACS Appl. Mater. Inter. 13 (25), 29439-29449. doi:10.1021/acsami.1c08565

Zhou, Q., Gallagher, R., Ufret-Vincenty, R., Li, X., Olson, E. N., and Wang, S. (2011). Regulation of Angiogenesis and Choroidal Neovascularization by Members of microRNA-23 2724 Clusters. Proc. Natl. Acad. Sci. 108 (20), 8287-8292. doi:10.1073/pnas.1105254108

Conflict of Interest: The authors declare that the research was conducted in the absence of any commercial or financial relationships that could be construed as a potential conflict of interest.

Publisher's Note: All claims expressed in this article are solely those of the authors and do not necessarily represent those of their affiliated organizations, or those of the publisher, the editors and the reviewers. Any product that may be evaluated in this article, or claim that may be made by its manufacturer, is not guaranteed or endorsed by the publisher.

Copyright (C) $2022 \mathrm{Wu}$, Qin, Wang, Yu, Liu, Peng, Liang, Zhang and Wei. This is an open-access article distributed under the terms of the Creative Commons Attribution License (CC BY). The use, distribution or reproduction in other forums is permitted, provided the original author(s) and the copyright owner(s) are credited and that the original publication in this journal is cited, in accordance with accepted academic practice. No use, distribution or reproduction is permitted which does not comply with these terms. 